\title{
RECENT DEVELOPMENTS ON THE F. AND M. RIESZ THEOREM
}

\author{
SHOZO KOSHI \\ Department of Mathematics, Faculty of Sciences, Hokkaido University \\ Sapporo 060, Japan
}

1. Introduction. In this note we describe some recent developments on the F. and M. Riesz theorem.

The original F. and M. Riesz theorem is as follows:

Let $\mathbb{T}=\{z:|z|=1\}=\left\{e^{i \theta}: 0 \leq \theta<2 \pi\right\}$ be the unit circle and $\mu$ be a complex Borel measure on $\mathbb{T}$ such that the Fourier transform of $\mu$ vanishes on the negative integers, i.e.

$$
\widehat{\mu}(n)=\frac{1}{2 \pi} \int_{\mathbb{T}} e^{-i n \theta} d \mu(\theta)=0 \quad \text { for } n=-1,-2, \ldots
$$

Then $\mu$ is absolutely continuous with respect to Lebesgue measure on $\mathbb{T}$.

From this theorem, we can deduce the famous Rudin-Carleson theorem: Let $F$ be a closed subset of $\mathbb{T}$. Every continuous function on $F$ can be extended to an analytic function inside the unit disk with boundary value on $F$ equal to the given continuous function on $F$ if and only if $F$ is a Lebesgue measure zero set.

Later, Helson and Lowdenslager generalized the F. and M. Riesz theorem to compact Abelian groups with ordered duals. De Leeuw and Glicksberg, Doss and Yamaguchi shortly afterwards obtained a number of related results.

2. Generalization of the F. and M. Riesz theorem to locally compact Abelian groups. Let $G$ be an Abelian group. We say that $G$ is an ordered group if $G$ contains a subsemigroup $P$ such that $P \cup(-P)=G$ and $P \cap(-P)=\{0\}$. We will call $P$ an order in $G$. It is well known that $G$ is an ordered group if and only if $G$ is torsion free. We shall write $x \geq y$ iff $x-y \in P$. It is easy to see that $\geq$ is a total order.

1991 Mathematics Subject Classification: 43A05, 43A25.

The paper is in final form and no version of it will be published elsewhere. 
Let $G$ be a locally compact Abelian group and let $\widehat{G}$ be its dual group. A fixed but arbitrary Haar measure on $G$ will be denoted by $m_{G}$. The symbol $M(G)$ will denote the Banach algebra of all bounded regular complex Borel measures on $G$ under convolution multiplication and the total variation norm. For an element $x$ in $G, \delta_{x}$ denotes the Dirac measure at $x$. For $\mu \in M(G)$, let $\mu_{a}$ and $\mu_{s}$ be respectively the absolutely continuous and singular parts of $\mu$ with respect to $m_{G}$. We denote the Fourier transform of a measure $\mu$ by $\widehat{\mu}$ and the convolution of measures $\mu$ and $\nu$ by $\mu * \nu$. For a subset $E$ of $\widehat{G}, M_{E}(G)$ denotes the space of measures in $M(G)$ whose Fourier transforms vanish on $\widehat{G} \backslash E$.

We now state the theorems mentioned in Section 1.

Theorem 1 (Helson-Lowdenslager). Let $G$ be a compact Abelian group with ordered dual $\widehat{G}$ and let $P$ be an order in $\widehat{G}$. If $\mu$ is a measure in $M_{P}(G)$ then $\mu_{a}$ and $\mu_{s}$ belong to $M_{P}(G)$ and moreover $\widehat{\mu}_{s}(0)=0$.

The proof of this theorem is found in [4] and based on a minimizing process in a certain Hilbert space.

Let $G$ be a compact Abelian group with dual $\widehat{G}$. It is known that there is an order in $\widehat{G}$ iff $G$ is connected.

If $G=\mathbb{T}^{2}$, then $\widehat{G}=\mathbb{Z}^{2}$ where $\mathbb{T}$ is the circle group and $\mathbb{Z}$ is the group of integers. $\mathbb{Z}^{2}$ is the set of lattice points $(m, n), m, n \in \mathbb{Z}$, in the Euclidean plane $\mathbb{R}^{2}$. An order in $\mathbb{Z}^{2}$ can be described by the half plane determined by a line $\ell: y=\alpha x$ through the origin 0 . If $\mathbb{Z}^{2} \cap \ell=\{0\}$, then $\alpha$ is irrational. If $\mathbb{Z}^{2} \cap \ell \neq\{0\}$, then $P \cap \ell$ is a half line and $\alpha$ is rational.

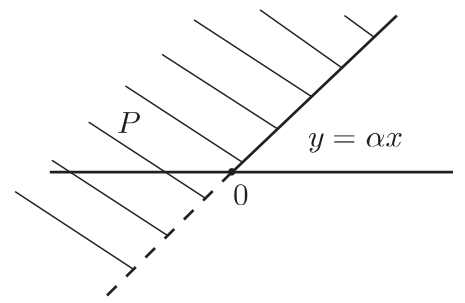

THEOREm 2. Let $G$ be a compact Abelian group and let $P$ be a subsemigroup of $\widehat{G}$ such that $P \cup(-P)=\widehat{G}$. If $\mu$ is a measure in $M_{P^{c}}(G)$, then $\mu_{a}$ and $\mu_{s}$ also belong to $M_{P^{c}}(G)$.

This theorem was first proved by Yamaguchi [15].

Using Theorem 2 we can prove the next theorem due to Doss:

Theorem 3 (Doss). Let $G$ be a locally compact Abelian group with ordered dual $\widehat{G}$ and let $P$ be an order in $\widehat{G}$. If $\mu$ is a measure in $M_{P}(G)$, then $\mu_{a}$ and $\mu_{s}$ belong to $M_{P}(G)$ and moreover $\widehat{\mu}_{s}(0)=0$.

Doss's proof of Theorem 3 has a flaw, since he tacitly assumes that an order $P$ is Haar measurable. We have found that there exists a non-Haar measurable order $P$ if we assume the axiom of choice. 
With the aid of Theorem 2, we can easily prove the following theorem due to Yamaguchi.

Theorem 4 (Yamaguchi). Let $G$ be a locally compact Abelian group and let $P$ be a subsemigroup of $\widehat{G}$ such that $P \cup(-P)=\widehat{G}$. If $\mu$ is a measure in $M_{P}(G)$, then $\mu_{a}$ and $\mu_{s}$ also belong to $M_{P}(G)$.

3. Characterization of groups with respect to the F. and M. Riesz theorem. If $G=\mathbb{T}^{2}$, then $\widehat{G}=\mathbb{Z}^{2}$ and $P=\{(m, n): n>0\} \cup\{(m, 0): m \geq 0\}$ is an order in $\mathbb{Z}^{2}$. If $\mu$ is the product measure $e^{i \theta} d \theta \times \delta_{0}$ on $\mathbb{T}^{2}$, then $\mu$ is singular with respect to the Haar measure on $\mathbb{T}^{2}$. We can easily show that $\mu$ is in $M_{P}\left(\mathbb{T}^{2}\right)$. Hence in $\mathbb{T}^{2}$ we cannot expect that every measure in $M_{P}\left(\mathbb{T}^{2}\right)$ is absolutely continuous with respect to the Haar measure.

Let $G$ be a locally compact group and $\widehat{G}$ be its dual group with a nondense order $P$ in $\widehat{G}$. We say that $P$ in $\widehat{G}$ has the FMR property if every measure in $M_{P}(G)$ is absolutely continuous. In other words, $P$ has the FMR property if $M_{P}(G) \subset L_{1}(G)$.

Examining the structure of orders in $\widehat{G}$, we get the following theorem:

Theorem 5. Let $A$ be a discrete divisible Abelian group, possibly $\{0\}$. Let $K$ be the character group of $A$ which is compact and torsion free. Let $G$ be the group $A \times \mathbb{T}$. Let $P$ be any nondense order in the dual group $K \times \mathbb{Z}=\widehat{G}$ of $G$. Then $P$ enjoys the FMR property.

Theorem 6. Suppose that $G$ is as in Theorem 5. Let $G$ be the group $\mathbb{R} \times A$. Let $P$ be an arbitrary nondense order in the dual group $\widehat{G}=\mathbb{R} \times K$ of $G$. Then $P$ enjoys the FMR property.

The converses of Theorems 5 and 6 are also true and we have the following theorem.

TheOREM 7. Suppose that $G$ is neither of the form $\mathbb{Z} \times K$ nor $\mathbb{R} \times K$ for a compact group $K$. Let $P$ be an arbitrary nondense order in the dual group $\widehat{G}$. Then there is a nonzero singular measure in $M_{P}(G)$.

Proofs of Theorems 5, 6, 7 are found in [5].

4. Glicksberg's theorem. Consider a subsemigroup $S$ of the dual group $\widehat{G}$ of a locally compact Abelian group $G$.

Glicksberg obtained the following theorem (an easy proof is given in [9]).

TheOREM 8. Let $G$ be a locally compact Abelian group and $\widehat{G}$ be its dual group. Let $S$ be a closed subsemigroup such that $S-S$ is dense in $\widehat{G}$. Then there exists a nonzero $\mu \in M_{S^{c}}(G)$ which is singular with respect to Haar measure $m_{G}$ on $G$ unless $G=\mathbb{R} \times \Delta$ or $\mathbb{T} \times \Delta$ for a discrete group $\Delta$.

The converse of Theorem 8 is also true. 
Theorem 9. Let $G$ and $S$ be as in Theorem 8. If $G=\mathbb{R} \times \Delta$ or $\mathbb{T} \times \Delta$ for a discrete group $\Delta$ and if $\mu \in M_{S^{c}}(G)$, then $\mu$ is absolutely continuous with respect to $m_{G}$.

$S$ is usually not an order in $\widehat{G}$ and Theorems 8 and 9 are new even in the case of $G=\mathbb{R}$.

In Theorems 8 and 9 , it is not necessary to assume the discrete group $\Delta$ to be divisible.

5. The F. and M. Riesz theorem in some convolution algebra in the unit disk. Let $D$ be the unit disk and $m$ be the positive measure on $D$ defined by

$$
d m(z)=\frac{\alpha+1}{\pi}\left(1-x^{2}-y^{2}\right)^{\alpha} d x d y,
$$

where $\alpha$ is a positive real number.

Let $M_{\alpha}(D)$ be the space of all bounded regular complex-valued Borel measures on $D$.

For each $z$ in $D$, the operator $T_{z}$ is defined by

$$
T_{z} f(\zeta)=\frac{\alpha}{\alpha+1} \int_{D} f\left(\bar{z} \zeta+\sqrt{1-|z|^{2}} \sqrt{1-|\zeta|^{2}} \xi\right) \frac{d m_{\alpha}(\xi)}{1-|\xi|^{2}}
$$

for $f$ continuous on $D$. By changing variables, if $z$ and $\zeta$ are in the interior of $D$, we find a function $E_{\alpha}(z, \zeta, \xi)$ with

$$
T_{z} f(\zeta)=\int_{D} f(\xi) E_{\alpha}(z, \zeta, \xi) d m_{\alpha}(\xi),
$$

where $E_{\alpha}(z, \zeta, \xi) \geq 0$ for $z, \zeta \in D^{\circ}, \xi \in D$ and

$$
\int_{D} E_{\alpha}(z, \zeta, \xi) d m_{\alpha}(\xi)=1 .
$$

If $\mu, \nu \in M(D)$, we define $\mu * \nu$ by

$$
\int_{D} f(t) d(\mu * \nu)(t)=\int_{D} \int_{D} T_{\bar{z}} f(\zeta) d \mu(z) d \nu(\zeta)
$$

for $f$ continuous on $D$. Then the new convolution operation $*$ makes $D$ a hypergroup, i.e. * is commutative and associative and $M_{\alpha}(D)$ is a commutative Banach algebra with unit under the multiplication operation $*$ and the usual total variation norm.

We define the Fourier transform $\widehat{\mu}$ of $\mu$ as a function of $(m, n) \in \mathbb{Z}^{+} \times \mathbb{Z}^{+}$, where $\mathbb{Z}^{+}$is the set of all nonnegative integers, as follows:

$$
\widehat{\mu}(m, n)=\int_{D} \bar{Q}_{m, n}^{\alpha}(z) d \mu(z),
$$


where

$$
Q_{m, n}^{\alpha}(z)=\frac{\alpha}{\alpha+1} \int_{D}\left(z+i \sqrt{1-|z|^{2}} \zeta\right)^{m}\left(\bar{z}+i \sqrt{1-|z|^{2}} \bar{\zeta}\right)^{n} \frac{d m_{\alpha}(z)}{1-|\zeta|^{2}} .
$$

After these preparations, we formulate the following F. and M. Riesz type theorem due to Kanjin [8].

Theorem 10 (Kanjin). Let $\alpha>0$ and $\mu \in M_{\alpha}(D)$. If there exists a positive integer $N$ such that $\widehat{\mu}(m, n)=0$ for $\min (m, n)>N$, then $\mu$ is absolutely continuous with respect to $m_{\alpha}$.

It is interesting that Theorem 10 can be extended to more general hypergroups.

\section{References}

[1] K. de Leeuw and I. Glicksberg, Quasi-invariance and analyticity of measures on compact groups, Acta Math. 109 (1963), 179-205.

[2] R. Doss, On measures with small transforms, Pacific J. Math. 26 (1968), 258-263.

[3] I. Glicksberg, The strong conclusion of the F. and M. Riesz theorem on groups, Trans. Amer. Math. Soc. 285 (1984), 235-240.

[4] H. Helson and D. Lowdenslager, Prediction theory and Fourier series in several variables, Acta Math. 99 (1958), 165-202.

[5] E. Hewitt and S. Koshi, Orderings in locally compact Abelian groups and the theorem of F. and M. Riesz, Math. Proc. Cambridge Philos. Soc. 94 (1983), 441-457.

[6] E. Hewitt and K. A. Ross, Abstract Harmonic Analysis, I, Grundlehren Math. Wiss. 115, Springer, Berlin, 1963.

[7] E. Hewitt, S. Koshi and Y. Takahashi, The F. and M. Riesz theorem revisited, Math. Scand. 60 (1987), 63-76.

[8] Y. Kanjin, A convolution measure algebra on the unit disc, Tôhoku Math. J. 28 (1976), $105-115$

[9] S. Koshi and Y. Takahashi, Generating subsemigroups, orders and a theorem of Glicksberg, Hokkaido Math. J. 16 (1987), 135-144.

[10] S. Koshi and H. Yamaguchi, The F. and M. Riesz theorem and group structures, ibid. 8 (1979), 294-299.

[11] F. Riesz und M. Riesz, Über die Randwerte einer analytischen Funktion, in: Comptes Rendus du 4. Congrès des mathématiciens scandinaves, Stockholm, 1916, 27-44.

[12] W. Rudin, Fourier Analysis on Groups, Wiley, New York, 1962.

[13] Y. Takahashi and H. Yamaguchi, On measures which are continuous by certain translation, Hokkaido Math. J. 13 (1984), 109-117.

[14] H. Yamaguchi, Remarks on Riesz sets, ibid. 7 (1978), 328-335.

[15] —, A property of some Fourier Stieltjes transform, Pacific J. Math. 108 (1983), 243-256. 